\title{
A randomized, controlled study evaluating effects of amlodipine addition to chelators to reduce iron loading in patients with thalassemia major
}

\author{
Bahador Bagheri ${ }^{1}$, Aziz Eghbali², Hamideh Kazemi², Yazdan Ghandi ${ }^{2}$, Mohammad Rafiei $^{3}$ \\ ${ }^{\text {I}}$ Pharmacology, Semnan University of Medical Sciences, Semnan, Iran, ${ }^{2}$ Department of Pediatrics, Arak University of \\ Medical Sciences, Arak, Iran, ${ }^{3}$ Department of Biostatistics, Arak University of Medical Sciences, Arak, Iran
}

Aim: Cardiomyopathy due to iron overload can be fatal in patients with thalassemia major. It has been shown by small number of studies that calcium channel blockers (CCBs) like amlodipine are able to reduce iron content of the heart. Our goal was to study effects of amlodipine addition to chelators on iron loading in patients with thalassemia major.

Methods: This randomized and controlled and single center trial was performed on 56 patients with thalassemia major in Amir Kabir Hospital (Arak-Iran). Sixty three patients were randomized to iron chelator and daily 2.5-5 mg amlodipine (Abidi Pharmaceutical-Iran) or iron chelator for one year. After one week of amlodipine therapy the dose increased to 5 mg. Iron content was measured by magnetic resonance imaging (heart $\mathrm{T}^{*}$ and liver $\mathrm{T} 2 *$ ). Serum ferritin was also measured. Trial registry was IRCT2015080720715N2.

Results: Differences in baseline characteristics were not significant. As shown in Figure, after 12 months of treatment myocardial T2* values had significant improvement in combined group $(\mathrm{P}<0.05)$. Difference between two groups was significant $(\mathrm{P}=0.02)$. Combined treatment had no effect on hepatic $\mathrm{T} 2^{*}$ value. Difference between two groups was not significant $(\mathrm{P}=0.2)$. There was a significant reduction in ferritin level at 12 months in the combined group $(\mathrm{P}<0.01)$. Mild gastrointestinal upset was the most common untoward effect.

Conclusion: Addition of amlodipine to iron chelators has beneficial effects for reduction of iron loading in patients with thalassemia major. This combination therapy seems safe. 